\title{
Neutrophil to lymphocyte ratio is an independent prognostic factor in patients with recurrent or metastatic head and neck squamous cell cancer
}

\author{
DERYA KIVRAK SALIM ${ }^{1}$, HASAN MUTLU ${ }^{1}$, MELEK KARAKURT ERYILMAZ ${ }^{1}$, OZAN SALIM ${ }^{2}$, \\ FATMA YALÇIN MUSRI ${ }^{1}$, DENIZ TURAL ${ }^{1}$, ŞEYDA GÜNDÜZ ${ }^{3}$ and HASAN ŞENOL COŞKUN ${ }^{1}$ \\ Departments of ${ }^{1}$ Medical Oncology and ${ }^{2}$ Hematology, Akdeniz University Hospital, School of Medicine, Antalya 07059; \\ ${ }^{3}$ Department of Medical Oncology, Antalya Training and Research Hospital, Antalya 07100, Turkey
}

Received September 25, 2014; Accepted March 4, 2015

DOI: $10.3892 / \mathrm{mco} .2015 .557$

\begin{abstract}
The neutrophil to lymphocyte ratio (NLR) has been widely investigated for its prognostic significance in cancer. In the present study, we aimed to determine whether NLR is a prognostic factor in patients with recurrent or metastatic head and neck squamous cell cancer (HNSCC). A total of 79 patients from the Akdeniz University database were retrospectively analyzed. The cut-off NLR was set at 2.93; patients with NLR $>2.93$ had a median overall survival (OS) of 12.1 months, whereas the median OS was not reached for patients with NLR $\leq 2.93$ ( $\mathrm{P}=0.027)$. On multivariate analysis, NLR and recurrence or metastatic site were found to be independent prognostic factors for $\mathrm{OS}(\mathrm{P}=0.014$ and $\mathrm{P}=0.002$, respectively). Therefore, NLR was identified as an independent prognostic factor for OS in patients with recurrent or metastatic HNSCC.
\end{abstract}

\section{Introduction}

Head and neck squamous cell carcinoma (HNSCC) is the sixth most common type of cancer worldwide (1), with an increasing trend in incidence. In 2014, 12,000 mortalities were expected to occur from head and neck cancer (2). Several factors affect survival and locoregional tumor control in patients with HNSCC (3-6). In order to better define the risks, analyses of prognostic factors and outcomes have been performed in a number of trials.

Systemic inflammation and immunity have recently been investigated in the context of HNSCC. The inflammatory response is crucial for the development and progression of this

Correspondence to: Dr Derya Kivrak Salim, Department of Medical Oncology, Akdeniz University Hospital, School of Medicine, Dumlupınar Boulevard, Kampüs, Antalya 07059, Turkey E-mail: deryakivrak@gmail.com

Key words: head and neck cancer, neutrophil to lymphocyte ratio, prognosis, survival type of cancer. The neutrophil to lymphocyte ratio (NLR) is a marker reflecting systemic inflammation and it may be easily calculated from the white blood cell counts. NLR was reported to be an independent prognostic factor for patients with various types of cancer. An increase in NLR has been shown to be associated with adverse overall survival (OS) in several solid tumors (3); a high NLR was associated with adverse outcomes of nasopharyngeal carcinoma, castration-resistant metastatic prostate carcinoma, breast cancer, non-small-cell lung cancer (NSCLC), local and advanced esophageal squamous carcinoma and renal cell carcinoma (7-14).

In the present study, we aimed to determine whether NLR is a prognostic factor for the survival of patients with recurrent or metastatic HNSCC.

\section{Patients and methods}

Patient characteristics. The clinicopathological characteristics and outcome data of 79 patients with recurrent or metastatic HNSCC were collected and retrospectively reviewed using the Akdeniz University database between 2003 and 2013. Age, gender, Eastern Cooperative Oncology Group performance status (ECOG PS), primary tumor site, stage, curative treatment protocol performed for local disease at diagnosis, induction chemotherapy, type of chemotherapy in the first-line setting and metastatic site at recurrence were imported into the Statistical Package for the Social Sciences software, version 16.0 (SPSS 16.0; SPSS, Inc., Chicago, IL, USA). Staging was performed according to the American Joint Committee on Cancer (AJCC) staging manual (15).

$N L R$. The neutrophil and lymphocyte counts at diagnosis of metastatic disease and at recurrence for local or locally advanced disease were recorded into SPSS 16.0. NLR was calculated as the ratio of absolute neutrophil count to absolute lymphocyte count in blood samples.

Survival. The date of diagnosis for patients with metastatic HNSCC or the date of recurrence for patients with recurrent HNSCC, time to progression and date of death of patients with recurrent and metastatic HNSCC were imported into the 
SPSS 16.0 statistical program. Progression-free survival (PFS) was defined as the time from treatment initiation to first evidence of disease progression. OS was defined as the time from treatment initiation to death from any cause.

Statistical analysis. Statistical analyses were performed using SPSS 16.0. To determine the characteristics of the patients, a frequency analysis was performed. The optimum cut-off value of NLR at recurrence or diagnosis was identified according to the receiver operating characteristic (ROC) curve and all 79 patients were divided into two groups based on the NLR value. According to the cut-off value of 2.93 , the effect of NLR on the PFS and OS of patients with metastatic or recurrent HNSCC was calculated by the log-rank test. Kaplan-Meier survival probability estimates were also calculated. A P-value of $<0.05$ was considered to indicate a statistically significant difference. The prognostic effect of NLR on survival (PFS and OS) in patients with recurrent or metastatic HNSCC was assessed by univariate and multivariate analyses.

\section{Results}

Patient characteristics. The characteristics of the two groups are summarized in Table I. The median age of the patients was 59 years (range, 28-85 years) and the majority of the patients were male (89.9\%). In terms of ECOG PS, $43 \%$ of the patients had a PS of $0,32.9 \%$ had $1,22.8 \%$ had 2 and $1.3 \%$ had 3. The most common primary tumor localization was the larynx. A total of 59 (74.6\%) patients had stage 4 disease, whereas $25.3 \%$ had local or locally advanced (stage I-II or III) disease. The most commonly used local treatment modality was surgery plus radiotherapy (39.2\%). In $16.5 \%$ of the patients, induction chemotherapy was performed. The most commonly used chemotherapeutic regimen $(64.6 \%)$ in the first-line setting was docetaxel $75 \mathrm{mg} / \mathrm{m}^{2}$ on day 1 , with cisplatin $75 \mathrm{mg} / \mathrm{m}^{2}$ on day 1 and 5 -fluorouracil $1,000 \mathrm{mg} / \mathrm{m}^{2}$ on days 1-5. Local or nodal recurrence was the most common recurrence site in patients with recurrent HNSCC.

$O S$ and PFS by NLR. Following ROC curve analysis, the optimum cut-off value of NLR was set at 2.93. The patients were divided into two groups, those with NLR $>2.93(n=44)$ and those with NLR $\leq 2.93$ ( $n=35)$. When the groups were evaluated in terms of PFS, there was no significant difference between the groups $(\mathrm{P}=0.109)$. However, the median OS was significantly higher in patients with NLR $\leq 2.93$ ( $\mathrm{P}=0.027$ ). The median PFS and OS values are presented in Table II. PFS and OS curves are shown in Figs. 1 and 2, respectively.

Survival analysis. Age, gender, primary tumor site, prior curative treatment protocol, prior induction chemotherapy, type of chemotherapy in the first-line setting, metastatic site at recurrence and NLR were evaluated in a univariate analysis regarding their effect on PFS and OS. As regards PFS, recurrence or metastatic site and prior induction chemotherapy were included in the multivariate analysis. Following Cox's regression analysis, only recurrence or metastatic site were identified as an independent factor affecting PFS $(\mathrm{P}=0.001)$ (data not shown).
Table I. Characteristics of the study patients $(\mathrm{n}=79)$.

\begin{tabular}{lc}
\hline Characteristics & $\begin{array}{c}\text { Patient no. }(\%) \\
(\mathrm{n}=79)\end{array}$ \\
\hline $\begin{array}{l}\text { Age, years [median (range)] } \\
\text { Gender }\end{array}$ & $59(28-85)$ \\
Female & $8(10.1)$ \\
Male & $71(89.9)$ \\
ECOG PS & \\
0 & $34(43.0)$ \\
1 & $26(32.9)$ \\
2 & $18(22.8)$ \\
3 & $1(1.3)$ \\
Primary tumor localization & \\
Larynx & $46(58.3)$ \\
Hypopharynx & $4(5.1)$ \\
Base of tongue & $9(11.4)$ \\
Tonsils & $5(6.3)$ \\
Paranasal or maxillary sinuses & $5(6.3)$ \\
Oral cavity & $2(2.5)$ \\
Other & $8(10.1)$
\end{tabular}

TNM stage at diagnosis

$\begin{array}{cc}1 & 1(1.3) \\ 2 & 3(3.8) \\ 3 & 16(20.2) \\ 4 & 59(74.7)\end{array}$

Local treatment modality

None

Radiotherapy

Surgery

Surgery + radiotherapy

Prior induction chemotherapy

Yes

No

Type of chemotherapy

$\mathrm{TCF}^{\mathrm{a}}$

$51(64.6)$

$\mathrm{CF}^{\mathrm{b}}+$ cetuximab

$19(24.0)$

Other

Recurrence site

Local or nodal

$35(44.3)$

Lung

Viscera other than lung

${ }^{\mathrm{a} D o c e t a x e l}+$ cisplatin + 5-fluorouracil. ${ }^{\mathrm{b}}$ Cisplatin + 5-fluorouracil ECOG PS, Eastern Cooperative Oncology Group performance status.

Following univariate analysis for OS, ECOG PS, recurrence or metastatic site, prior induction chemotherapy and NLR were included in the multivariate analysis $(\mathrm{P}=0.138$, $\mathrm{P}=0.004, \mathrm{P}=0.128$ and $\mathrm{P}=0.061$, respectively) (data not shown). Following Cox's regression analysis, only NLR and recurrence or metastatic site were found to be independent significant prognostic factors affecting $\mathrm{OS}(\mathrm{P}=0.014$ and $\mathrm{P}=0.002$, respectively) (data not shown). 
Table II. Survival analysis.

\begin{tabular}{lccr}
\hline Median survival (months) & NLR $>2.93(\mathrm{n}=44)$ & NLR $\leq 2.93(\mathrm{n}=35)$ & P-value \\
\hline PFS & $6.34(95 \%$ CI 3.55-9.12) & $9.19(95 \%$ CI 1.53-16.8) & 0.109 \\
OS & $12.1(95 \%$ CI 9.75-14.4) & NR & 0.027
\end{tabular}

NLR, neutrophil to lymphocyte ratio; PFS, progression-free survival; OS, overall survival; CI, confidence interval; NR, not reached.

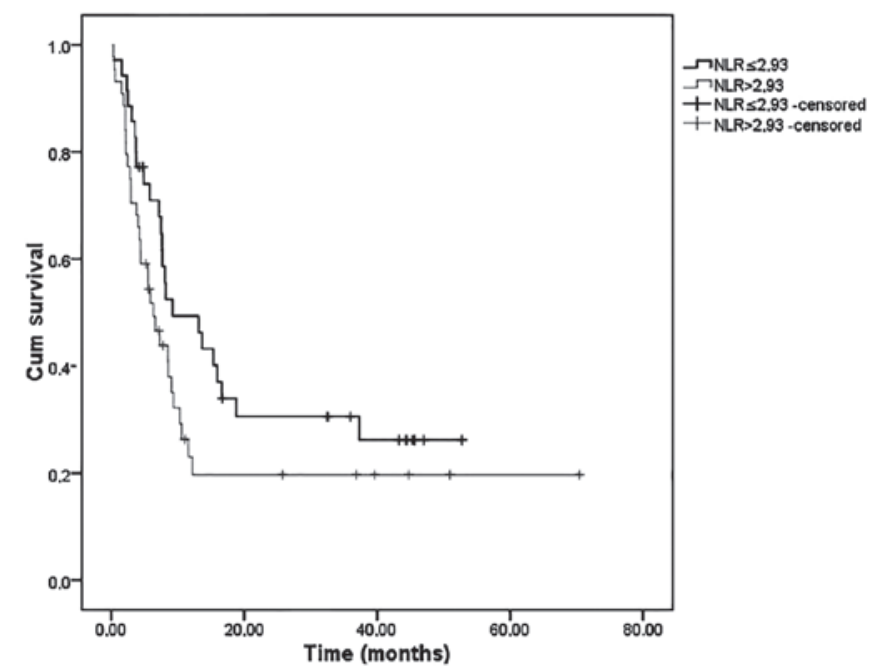

Figure 1. Progression-free survival curves for patients with neutrophil to lymphocyte ratio (NLR) $>2.93$ and $\leq 2.93$ ( $\mathrm{P}=0.109)$. Cum, cumulative.

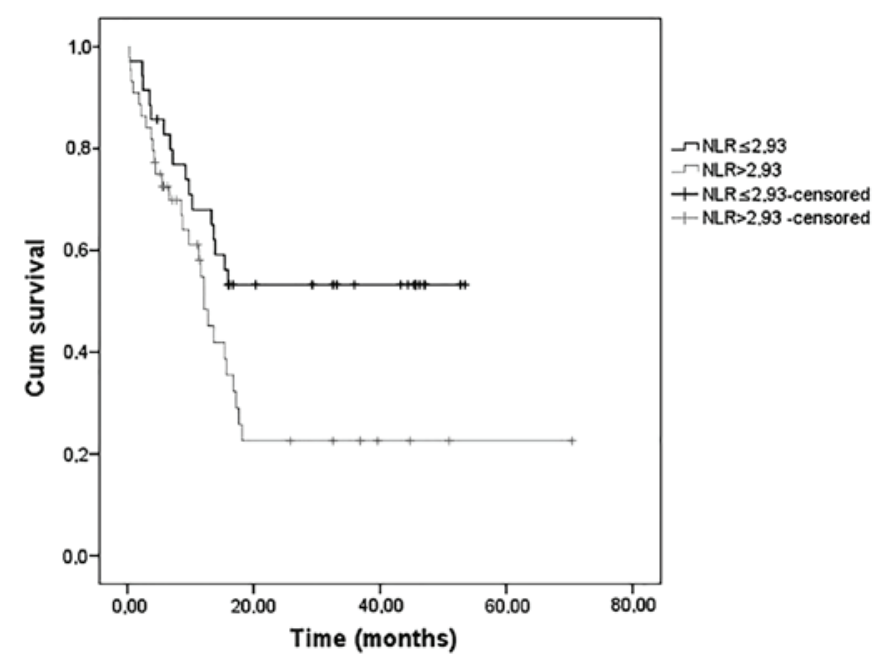

Figure 2. Overall survival curves for patients with neutrophil to lymphocyte ratio $(\mathrm{NLR})>2.93$ and $\leq 2.93(\mathrm{P}=0.027)$. Cum, cumulative.

\section{Discussion}

In the present study, we investigated the prognostic effect of NLR on OS and PFS in recurrent or metastatic HNSCC. Our results revealed that NLR is an independent significant prognostic factor for OS $(\mathrm{P}=0.002)$ in patients with recurrent or metastatic HNSCC.

Cancer-related inflammation has been shown to negatively affect cancer prognosis. Neutrophilia may be a marker of inflammation-related aggressive biological behaviour of the tumor (18). Absolute neutrophil and lymphocyte counts may be affected by various physiological, pathological and physical factors, although NLR may remain stable with respect to these factors. Therefore, NLR may be prognostically superior to leukocyte subtype and high NLR values resulting from inflammation are considered to be associated with poor prognosis (16). The exact association of high NLR and poor outcome in cancer patients has not been fully elucidated. Neutrophilia may inhibit the immune system by suppressing lymphocytes, activated $\mathrm{T}$ cells and natural killer cells $(3,17)$. The prognostic role of the NLR has been evaluated in $>60$ studies on a number of solid tumors $(7-14,16,18)$.

In a systemic review analyzing cancer-specific survival, a high NLR was found to be associated with worse OS (3). A retrospective review of patients with esophageal squamous cell carcinoma demonstrated that a preoperative NLR of $\geq 2.5$ may be predictive of poor prognosis following radical resection. In the same study, NLR was found to be an independent prognostic risk factor (12). In another retrospective study, NLR was suggested to be a predictor of chemosensitivity in advanced esophageal cancer patients receiving neoadjuvant chemotherapy (10). Wang et al (11) demonstrated that elevated NLR at recurrence of NSCLC indicates poor prognosis and suggested it may be a significant independent prognostic factor in patients with recurrent NSCLC following curative resection. Nakano et al (9) investigated the association between NLR and survival outcome in preoperative local and locally advanced breast cancer and concluded that it may be an independent prognostic factor for disease-free survival and breast cancer-specific survival in patients with breast cancer. It has also been demonstrated that increased pretreatment NLR is an independent prognostic factor in patients with metastatic renal cell carcinoma who received tyrosine kinase inhibitors (14).

When evaluating the association between NLR and HNSCC, it was previously demonstrated that high pretreatment peripheral NLR was significantly associated with poor PFS among patients with advanced clinical stage (III and IV) nasopharyngeal carcinoma (7). However, there was no significant association between NLR and PFS in the present study. Apart from the limited number of studies on nasopharyngeal carcinoma, there are no studies in the literature demonstrating the prognostic significance of NLR in head and neck carcinomas, mainly laryngeal carcinoma. In another study on oral cavity cancer patients, it was observed that NLR increased in parallel with the advancement of clinical stage and $\mathrm{T}$ stage, but it was not significantly associated with survival (18). Furthermore, the opposite finding has also been reported, namely better survival associated with higher 
NLR in HNSCC patients, suggesting an antitumorigenic role of NLR (19). Rassouli et al (20) reported that NLR $\geq 4.2$ predicts a higher recurrence rate $(\mathrm{P}<0.0001, \log$-rank test $)$ and concluded that NLR may be used as an independent predictor of recurrence and survival. A retrospective study on pretreatment NLR and disease-spesific survival in patients with oral cancer undergoing preoperative chemoradiotherapy indicated that NLR is a significant independent predictor of poor cancer-specific survival (21). In accordance with the literature regarding cancer types other than HNSCC, our results also identified NLR as an independent prognostic factor in patients with recurrent or metastatic HNSCC.

NLR derived from a single blood sample at initial diagnosis of metastatic disease or at recurrence may be a useful laboratory marker for recurrent or metastatic HNSCC. This simple, cost-effective, non-invasive and rapidly available test may be easily used in clinical practice.

Combined with other markers, NLR may be used in decision-making and the selection of treatment modality in patients with recurrent or metastatic HNSCC. Although the present study was a retrospective, single-center study, it indicates the potential benefit of a novel prognostic marker in HNSCC. However, our findings require confirmation by larger, prospective, randomized studies.

\section{References}

1. Hunter KD, Parkinson EK and Harrison PR: Profiling early head and neck cancer. Nat Rev Cancer 5: 127-135, 2005.

2. Siegel R, Ma J, Zou Z and Jemal A: Cancer statistics, 2014. CA Cancer J Clin 64: 9-29, 2014.

3. Templeton AJ, McNamara MG, Šeruga B, et al: Prognostic role of neutrophil-to-lymphocyte ratio in solid tumors: a systematic review and meta-analysis. J Natl Cancer Inst 106: dju124, 2014.

4. Huang AT, Tang C, Bell D, et al: Prognostic factors in adenocarcinoma of salivary glands. Oral Oncol: Apr 2, 2015 (Epub ahead of print).

5. Iyer NG, Dogan S, Palmer F, et al: Detailed analysis of clinicopathologic factors demonstrate distinct difference in outcome and prognostic factors between surgically treated HPV-positive and negative oropharyngeal cancer. Ann Surg Oncol: Mar 24, 2015 (Epub ahead of print)

6. Krestevska V, Stojkovski I, Zafirova-Ivanovska B and Crvenkova S: Prognostic factors in patients with advanced hypopharyngeal squamous cell carcinoma treated with concurrent chemoradiotherapy. J BUON 17: 327-336, 2012.

7. He JR, Shen GP, Ren ZF, et al: Pretreatment levels of peripheral neutrophils and lymphocytes as independent prognostic factors in patients with nasopharyngeal carcinoma. Head Neck 34 $1769-1776,2012$.
8. Templeton AJ, Pezaro C, Omlin A, et al: Simple prognostic score for metastatic castration-resistant prostate cancer with incorporation of neutrophil-to-lymphocyte ratio. Cancer: July 3, 2014 (Epub ahead of print)

9. Nakano K, Hosoda M, Yamamoto M and Yamashita H: Prognostic significance of pre-treatment neutrophil:lymphocyte ratio in Japanese patients with breast cancer. Anticancer Res 34: 3819-3824, 2014.

10. Sato H, Tsubosa Y and Kawano T: Correlation between the pretherapeutic neutrophil to lymphocyte ratio and the pathologic response to neoadjuvant chemotherapy in patients with advanced esophageal cancer. World J Surg 36: 617-622, 2012.

11. Wang X and Li K: Neutrophil-to-lymphocyte ratio predicts the survival in patients with post-operative recurrence of non-small cell lung cancer. Chin J Oncol 36: 298-302, 2014 (In Chinese).

12. Chen $\mathrm{H}$ and $\mathrm{He} \mathrm{J}$ : Preoperative neutrophil-to-lymphocyte ratio as a prognostic predictor after radical resection of esophageal squamous cell carcinoma. Chin J Oncol 36: 294-297, 2014 (In Chinese).

13. Yoo EJ, Park JC, Kim EH, et al: Prognostic value of neutrophil-to-lymphocyte ratio in patients treated with concurrent chemoradiotherapy for locally advanced oesophageal cancer. Dig Liver Dis 46: 846-853, 2014.

14. Gunduz S, Mutlu H, Uysal M, Coskun HS and Bozcuk H: Prognostic value of hematologic parameters in patients with metastatic renal cell carcinoma using tyrosine kinase inhibitors. Asian Pac J Cancer Prev 15: 3801-3804, 2014.

15. Edge S, Byrd DR, Compton CC, et al (eds): AJCC Cancer Staging Manual. 7th edition. Springer, New York, NY, pp41-113, 2010.

16. Dirican A, Kucukzeybek BB, Alacacioglu A, et al: Do the derived neutrophil to lymphocyte ratio and the neutrophil to lymphocyte ratio predict prognosis in breast cancer? Int J Clin Oncol: February 18, 2014 (Epub ahead of print).

17. el-Hag A and Clark RA: Immunosuppression by activated human neutrophils. Dependence on the myeloperoxidase system. J Immunol 139: 2406-2413, 1987.

18. Tsai YD, Wang CP, Chen CY, et al: Pretreatment circulating monocyte count associated with poor prognosis in patients with oral cavity cancer. Head Neck 36: 947-953, 2014.

19. Millrud CR, Månsson Kvarnhammar A, Uddman R, Björnsson S, Riesbeck K and Cardell LO: The activation pattern of blood leukocytes in head and neck squamous cell carcinoma is correlated to survival. PLoS One 7: e51120, 2012.

20. Rassouli A, Saliba J, Castano R, Hier M and Zeitouni AG: Systemic inflammatory markers as independent prognosticators of head and neck squamous cell carcinoma. Head Neck: December 13, 2013 (Epub ahead of print).

21. Perisanidis C, Kornek G, Pöschl PW, Holzinger D, Pirklbauer K, Schopper C and Ewers R: High neutrophil-to-lymphocyte ratio is an independent marker of poor disease-specific survival in patients with oral cancer. Med Oncol 30: 334, 2013. 